\title{
Urgences
}

\section{Sur trois notes}

\section{Élisabeth Haghebaert}

Numéro 31, mars 1991

Poétique de la note

URI : https://id.erudit.org/iderudit/025635ar

DOI : https://doi.org/10.7202/025635ar

Aller au sommaire du numéro

Éditeur(s)

Urgences

ISSN

0226-9554 (imprimé)

1927-3924 (numérique)

Découvrir la revue

Citer cet article

Haghebaert, É. (1991). Sur trois notes. Urgences, (31), 44-55.

https://doi.org/10.7202/025635ar

Ce document est protégé par la loi sur le droit d'auteur. L'utilisation des services d'Érudit (y compris la reproduction) est assujettie à sa politique d'utilisation que vous pouvez consulter en ligne.

https://apropos.erudit.org/fr/usagers/politique-dutilisation/
Cet article est diffusé et préservé par Érudit.

Érudit est un consortium interuniversitaire sans but lucratif composé de l’Université de Montréal, l'Université Laval et l'Université du Québec à Montréal. Il a pour mission la promotion et la valorisation de la recherche. https://www.erudit.org/fr/ 


\title{
Sur trois notes Élisabeth Haghebaert
}

\begin{abstract}
On me pardonnera si je collectionne, pour la lucidité, ici tels débris au coupant vif, omissions, conséquences, ou les regards inexprimés. Ce sera ces Notes.
\end{abstract}

\section{Mallarmé, La musique et les lettres}

Statutairement précaire, subordonnée, ambivalente et généralement controversée, sinon vilipendée ${ }^{1}$, la note $\mathbf{a}-$ on le sait - un rôle ingrat. A jouer les sempiternelles utilités, elle ne serait, par rapport au fragment par exemple, qu'un disgracieux sous-produit. Si l'on en juge d'un côté par la réticence ou la condescendance que suscitent son étude ${ }^{2}$ et les discussions terminologiques ${ }^{3}$ qu'elle provoque, de l'autre par certaines pratiques qui se manifestent en littérature pensons à l'emploi qu'en font Genet, Jamek ou Le Clézio, de manière relativement subreptice il est vrai -, la ou les questions qu'elle soulève ouvrent à l'analyse un territoire prometteur.

Les notes de lecture qui suivent donnent en trois exemples un aperçu de l'usage de la note dans le texte autobiographique et dans le texte de fiction qui permettra peutêtre de mesurer la ténuité de la distinction entre les deux. Quel effet l'usage d'un additif textuel tel que la note peut-il exercer dans la fiction? Telle est la question que pose entre autres l'exemple proposé par Le Clézio. Quelles sont les

\footnotetext{
1 G. W. Bowerstock, "The art of the footnote", The American Scholar, Washington, vol. 53, $n^{\circ} 1,1983-1984$, p. 59: «In England and America a superabundance of bulky notes has invariably seemed a Teutonic weakness ".

2 Pour un panorama bibliographique des travaux sur le sujet, voir Gérard Genette, Seuils, coll. “ Poétique”, Paris, Seuil, 1987, p. 293-315 (* Les notes "), et Jan Baetens, "La question des notes: l'exemple de Jean Ricardou ", Rivista di letterature moderne e comparate, Pise, Pacini Editore, vol. XL, fasc. 4, 1987, p. 357-367.

3 Je pense en particulier à la distinction entre périgraphie et paratexte, explorée par Jan Baetens dans "Bande à part: sur la pratique de la bandeannonce", Conséquences, Paris, $n^{\circ} 1,1983$, p. 66-79.
} 
limites de la note? Où commence-t-elle, où finit-elle, que définit-elle? Voilà ce qu'éprouvent les textes de Genet et de Jamek ${ }^{4}$.

Cet usage des notes en littérature, implicitement situé en marge des conventions, est en effet tout sauf innocent. Expédient discursif ou intrusion parasitaire dans le déroulement du texte, il offre une voie d'accès à un programme d'écriture. Même si on exempte a priori celui-ci d'un parti pris systématique de rébellion ou de contestation, le soupçon pèse sur lui. On ne peut dénier que l'apparition subite de la note dérange, à plus forte raison quand elle agit comme texte. Le caractère imprévisible du moment où elle survient, ainsi que la prolifération et l'expansion difficiles à juguler dont elle peut faire preuve ajoutent aux facteurs d'irritabilité qu'elle produit parfois chez le lecteur. En retour, sous prétexte de fournir l'information indispensable à la compréhension du texte qui l'engendre, elle oppose, imparable, son statut facultatif.

Bref, en perturbant l'ordre établi, la note impose la réflexion sur le choix du processus lectoral que sollicite l'œuvre. Si l'on adhère à l'idée que les facilités offertes par le traitement de texte réactivent et banalisent l'emploi de la note tout en le modifiant - telle précision tardive pouvant maintenant facilement s'insérer et s'intégrer dans le texte l'“appareil" que celle-ci met à la disposition de l'écrivain est bel et bien un outil susceptible de dynamiser, pour le meilleur et pour le pire, le trio lecteur-texte-auteur.

En marge d'exemples extrêmes, plus représentatifs ou plus conformes peut-être à certain anticonformisme, la sélection définie se propose avant tout de montrer que la recrudescente présence de cette forme ancienne constitue un enjeu non dépourvu d'intérêt dans la littérature actuelle.

Aux notes infrapaginales avec appel chiffré chez Le Clézio et Genet, à la note avec astérisque, insérée dans le texte chez Jamek, il faudrait encore en effet ajouter à titre d'échantillonnage de ce que l'on rencontre dans la littérature récente les

4 Jean-Marie G. Le Clézio, Le livre des fuites, roman d'aventures, Paris, Gallimard, 1969, p. 191; Jean Genet, Fragments... et autres textes, Paris, Gallimard, 1990, p. 69-97; Vaclav Jamek, Traité des courtes merveilles, Paris, Grasset, 1989, p. 15, 16, 31, 33, 43, 46, 51, 59, 70, 91, 95, 106, 108, 111, 141, $147,161,188,197,226,252,272,298$. 
«Note» et «digression » désignées comme telles chez Ransmayr et Quignard ${ }^{5}$. Ceci pour donner un aperçu de la diversité de formes ${ }^{6}$ que revêt la note et rappeler la difficulté à laquelle se heurte la plus modeste tentative de typologie. Tous ces exemples mettent en effet en évidence le caractère banal et usuel mais difficile à cerner - «élusif " et " fuyant» - de ce que Genette définit comme un «énoncé de longueur variable relatif à un segment plus ou moins déterminé du texte et disposé soit en regard soit en référence à ce segment" 7 .

\title{
Note 1
}

Fictionnelle mais ambiguë est la note que l'on trouve en page 191 du Livre des fuites ici reproduite:

\section{Autocritique}

II faut que je me décide à prendre enfin les résolutions suivantes:
a) Dire tout ce que je pense
b) Renoncer aux mots qui font plaisir
c) Ne pas essayer de tout faire à la fois ${ }^{1}$
d) Ne plus avoir peur des noms
e) Changer de marque de crayon à bille

\begin{abstract}
1. A moins qu'il ne s'agisse, au contraire, de tout dire en même temps. La litterature n'est-elle pas, en ce cas (et particulièrement la a fiction *), l'effort désesperé et permanentement mis en échec pour produire une expression unique? Dans le genre d'un cri peut-étre, d'un cri qui contiendrait de façon inexplicable les millions de mots de tous les temps et de tous les lieux. Contrairement a la parole qui classe, l'ecriture ne chercherait-elle que l'couf, le germe?
\end{abstract}

À la fictionnalité près, le régime de cette note est celui de la note infrapaginale ordinaire. Sa particularité est d'être unique dans le livre et, semble-t-il, dans l'œuvre romanesque de Le Clézio. Bien que celui-ci utilise fréquemment dans ses premiers livres la variété des ressources typographiques para- et extratextuelles, celle-ci reste inusitée. Appel chiffré, taille des caractères, situation en bas de page: tout est ici d'une conformité si parfaite au conventionnel qu'est instantanément convoqué le soupçon de simulacre satirique.

5 Christoph Ransmayr, Les effrois de la glace et des ténèbres, Paris, Maren Sell, 1989, p. 41; Pascal Quignard, Albucius, Paris, P.O.L. , 1990, p. 72.

6 La combinatoire des possibles en ce domaine, en particulier en ce qui concerne la disposition, est généreusement évoquée par Genette dans Seuils, p. 294-295.

7 Seuils, p. 293. 
Attribuée au personnage narrateur en charge du "roman d'aventures" dont un certain J.H.H. (Jeune Homme Hogan) est le héros ou au moins le fil conducteur, elle conferre à ce narrateur une fonction auctoriale. Celle-ci est d'autant plus vraisemblable qu'elle s'insère dans un des sept ou huit relais du texte rédigés à la première personne et intitulés "autocritique». (Faut-il préciser que ce "roman d'aventures" se révèle aussi roman d'initiation?) L'intention est semble-t-il sans équivoque puisque la simple conjonction du titre et de la note avec son appel suffit à créer cet effet de véridiction. La ressemblance aidant, cette conjonction instaure même un continuum avec le monde extrafictionnel auquel le lecteur est libre d'adhérer ou non. La confusion entre narrateur et auteur s'accomplit en pleine fiction de nonfiction. Pourquoi pas, en effet, imaginer comme plausible le contenu de cette note dans le carnet d'un jeune écrivain? N'était que cela pourtant, cet exemple si intéressant soit-il dans son minimalisme ne porterait pas à développement.

Lintérêt réel de cette note réside dans la relation qu'elle entretient avec le texte auquel elle se rapporte. Celui-ci, squelettique - il s'agit d'une énumération - énonce les cinq «résolutions» du narrateur, alias le pseudo-romancier réel. D'ordre général, la troisième de ces résolutions ne prend sens qu'à la lumière du contenu de la note qu'elle a engendrée. Contrastant avec le style télégraphique du "texte" générateur (une ligne), cette note - sorte de fragment de journal ou de note à usage personnel -, six foix plus longue que le texte de départ, amorce sous forme de deux questions au style direct une réflexion métaphorique sur la littérature et le sens de l'écriture, en particulier le rapport de la parole à l'écriture. À ce stade, deux constats s'imposent.

1) Tout en participant à la fictionnalité, la note pervertit celle-ci dans la mesure où le contenu de l'interrogation qu'elle transmet concerne la fabrique même du roman. Tout se passe comme si, contrairement à l'usage en vigueur, l'usage de la note autorisait à expurger le texte en convoquant à sa place la réflexion métatextuelle. Désignée par Karlheinz Stierle ${ }^{8}$ comme "lieu privilégié de la dimension pragmatique", la note permet en effet, le temps d'un raccourci préconceptuel, de reléguer la fiction au rang d'illusion et d'exercer ainsi une fonction supplétive. Manœuvre spéculaire s'il en est, puisque 
la simulation de la note fait partie du texte qui se regarde en train de se constituer. De même, les "questions" fondamentales se posent, concrètement, à la forme interrogative. En fait le contretexte ${ }^{9}$ ainsi instauré renverse le cours du texte initial ou en tout cas principal (si l'on se fie à la taille des caractères), de sorte que l'artifice tactique se mue en lieu réflexif contestataire.

En ce sens, la relation note-texte est ici réductible à un exemple de dénégation - celle-ci, rappelons-le, étant le "procédé par lequel le sujet tout en formulant un de ses désirs, pensées, sentiments jusqu'ici refoulé continue à s'en défendre en niant qu'il lui appartienne" ${ }^{10}$. Emblème traditionnel de la communication savante, la note contribue en effet simultanément - par le détour du pastiche - à sa propre mise en cause et au renforcement de sa crédibilité. Or la note n'est-elle pas également, par tradition, le refuge le plus sûr pour les hypothèses les plus audacieuses? Le lieu du non-lieu où, dédouané de tout sentiment de culpabilité, l'auteur sans contraintes ni dommages peut, s'il le veut, se livrer aux élucubrations les plus subversives?

2) Remettant en cause l'univers textuel auquel elle appartient, cette note attire l'attention sur les multiples possibles du texte. Bien qu'articulée à une histoire fictive, elle semble convoquer un champ de référence d'un degré de fictionnalité moindre. En effet, l'artifice de la dimension pseudo autocritique du texte auquel elle se rattache ne parvient pas à dissimuler que la forme interrogative directe utilisée dans son corps sollicite la participation du lecteur, ce qui n'arriverait pas avec le classique (et objectif) appareil de notes critiques.

Cette intervention, qui met l'accent sur la fonction phatique du discours, impose ainsi de nouvelles limites à la fiction en incluant le narrateur et le lecteur. Pratiquée d'abondance au XVIII siècle, en particulier dans les préfaces, cette interpellation trouve par ailleurs sa justification dans l'intertexte leclézien même. Il suffit de relire la préface

9 Au sens où, d'après Pierre Bec (cité dans Urgences, Rimouski, $n^{\circ} 19$, janvier 1988, p. 49-50), " Le code textuel [...] fonctionne toujours dans la plénitude de ses moyens mais à contre-courant ".

10 J. Laplanche et J.-B. Pontalis, Vocabulaire de la psychanalyse, Paris, PUF, 1967, p. 112. 
auctoriale du Procès-verbal pour se convaincre d'une relation de continuité entre cette note et non seulement le texte qui la précède immédiatement mais également le tout premier texte de l'œuvre: «Il me semble qu'il y a [...] d'énormes espaces vierges à prospecter, d'immenses régions gelées s'étendant entre auteur et lecteur [...] Ȧ mon sens, écrire et communiquer, c'est être capable de faire croire n'importe quoi à n'importe qui " ${ }^{11}$. Ceci rappelle que, autant que toute autre, la lecture de la note en bas de page implique d'avoir en mémoire la multitextualité inhérente à tous les textes ${ }^{12}$. En ce sens également, produit d'un acte créatif, cette note unique et déstabilisatrice cautionne sa propre fonction herméneutique au sein de l'œuvre en procurant au lecteur l'occasion de considérer la narration comme lieu d'incertitude et de mise en doute.

\section{Note 2}

Le texte en deux parties de Genet intitulé "Fragments..." 13 , selon la description d'Edmund White, "use de notes en bas de pages, de passages décalés et imprimés en caractères plus petits, d'espacements fréquents entre les paragraphes, d'intertitres et de marginalia pour produire une inversion du sens de la subordination et un effondrement de la hiérarchie des éléments littéraires ". De même source, on apprend que cette disposition, adoptée plus tard dans la publication aux éditions Gallimard de $\mathrm{Ce}$ qui est resté d'un Rembrandt déchiré en petits carrés..., sera utilisée par Derrida pour Glas (1974). De fait, comme l'indique la note auctoriale en exergue:

Les pages qui vont suivre ne sont pas extraites d'un poème: elles devraient y conduire. C'en serait l'approche, encore très lointaine, s'il ne s'agissait d'un des nombreux brouillons d'un texte qui sera démarche lente, mesurée, vers le poème, justification de ce texte comme le texte le sera de ma vie.

Ce texte en prose destiné à devenir poème constitue, à partir de la conjugaison d'éléments autobiographiques ainsi que de

11 J.M.G. Le Clézio, Le procès-verbal [1963], coll. "Folio" n 353, Paris, Gallimard, 1984, p. 11.

12 D'après Shari Benstock, At the margin of the discourse: Footnotes in the fictional text ", PMLA, New York, vol. 98, n², mars 1983, p. 204-225.

13 Paru pour la première fois dans la revue Les Temps modernes, Paris, $n^{\circ} 105$, août 1954, et repris tout récemment dans le recueil intitulé Fragments... et autres textes. 
50

réflexions esthétiques et éthiques traitant de l'homosexualité, un projet d'écriture de type mallarméen.

Dans le premier *fragment d'un discours", dix notes, classiquement numérotées et de longueur variable apparaissent en bas de page. Dans le second «fragment" (p. 92-93), les notes, sans appel, occupent généralement l'espace vertical droit d'une page partagée avec un dialogue, et le texte qui les compose et s'y substitue ne doit son apparence de note qu'à la taille de ses caractères et à la proximité des autres notes.

Quel que soit le contenu de chacune d'elles, variable autant que leur disposition, toujours surprenant est le moment où elles sont convoquées. Imprévisible pour le lecteur, ce moment apparemment arbitraire obéit aux lois d'une logique idiosyncrasique qui, on l'imagine en regard des prémisses, se fonde sur l'autobiographie.

L'intérêt de ce texte en regard de l'usage des notes vient de ce qu'il donne la parole à différents degrés d'énonciation allant du strict commentaire à la théâtralisation - qui correspondent aux éléments d'un triptyque où coexistent l'évocation de la jouissance sensuelle, la distanciation propre à la contemplation esthétique, avant la dissociation totale qui correspond à l'attitude théorique. Ce qui frappe dans la relation texte-notes, autant que l'écart entre expression et contenu, ce sont en effet les variations de ton et la tension qui en résulte.

Si l'on en croit la note liminaire non numérotée, dans le contexte d'esquisse qui est celui du projet d'ensemble, la note dédouble le texte, ainsi soumis à une double partition. Tantôt en sourdine, neutre et rationnelle, tantôt lyrique, pour faire chorus, elle multiplie concrètement et consciemment ses possibles en appelant autant aux affects et aux sens qu'à la raison, ainsi: "Les vocables tiennent aussi leurs pouvoirs de ces puissances qui les sacrent, et auxquelles ils renvoient, mais qui donneraient tant de pouvoir aux puissants s'ils ne se référaient à un ordre qui fut sacré par le chant " (extrait de la note 1, p. 82). En ce sens, la note, sortant de la légendaire sobriété où elle se cantonne d'ordinaire, s'inscrit dans une logique d'effusion et de profusion où s'exalte le texte.

Par exemple, comme s'il en évacuait le trop-plein, le lyrisme sensuel et réaliste de la première note corrobore le texte dont par une sorte d'effet démultiplicateur il élargit la 
perspective sans exclure la possibilité de dialogue entre le texte " officiel» et le texte de la note. L'alternative que pose la note établit en effet une nouvelle figure dans la narration, parallèle à la première:

La paupière morose - où la chimère est frappée, tu guettais '. Mais, de mes ténèbres miraculeusement décroché, pour mes draps, te voici venir me lécher du dehors, naîf encore, hésitant entre: le gamin et le jeune cavalier, la fille et le soleil, la rose et le garçon, la lune et la mort - chaque fois à la seconde d'une autre métamorphose - la mort et ce livre. À qui sinon toi, parler de toi afin d'instaurer - jusqu'à la ruine équitable, d'échos toujours plus sourds - un dialogue inutile? Sur ta personne voici les pires détails. Réfugie-toi dans l'horreur de ce texte d'abord, dans notre confusion ensuite, puis dans une région solitaire, hors d'atteinte, la Légende, si tu l'oses. [...]

1. "Etranges amours! Une crépusculaire odeur vous isole. Cependant c'est moins le monstre dépeigné de vos corps s'emboltant qúe son image multipliée dans les glaces d'un bordel - ou votre délicat cerveau? - qui vous trouble! En nage vous remontez de ces contrées absurdement lointaines: vous aviez chaviré en vous-méme où la fuite est plus súre, votre ivresse s'y gonflant jusqu'à l'explosion - de votre seule et réciproque exhalaison. Nommez amours ces jeux de reflets qui s'épuisent, s'époumonent à n'en plus finir sur les murs des chambres dorées..

Ainsi parle une oblique raison qui regarde, fascinée, dans chaque accident la mort apparaitre. [...] (p. 70 )

La note en modulant le texte le redouble comme par effet de miroir. Le contraste et l'effet de surprise qui en résultent sont d'autant plus grands lorsque contenu et ton s'intellectualisent et théorisent: "Je n'ignore plus que d'un fait singulier impuissant à conduire à une morale, on doit tirer, si l'on est cohérent, une esthétique " (note 1, p. 79).

Aucune limite obéissant à la logique commune qui sépare, classe et hiérarchise, ne semble intervenir entre le narrateur ou l'auteur et son dire, toute prédestination formelle bannie. Ce qui se trouve dans les notes pourrait figurer dans le texte et vice-versa, comme si une absence de censure effaçait en dépit des apparences toute distinction de registre et de genre. Située dans le champ de l'excès et du débordement, la note refuse ici cependant le statut structurel d'exutoire et, en revendiquant un statut polymorphe et labile, illustre a contrario le lien rhétorique qui maintient le langage dans les marges du discours. Contrairement au schème situant l'explication dans la note, peut-être est-ce, via le texte même, dans la dimension métatextuelle et dans l'adéquation de la 
52

forme au projet qu'il faut chercher une explication. On verrait ainsi que telle mesure est aussi soumise au régime de la dénégation:

Enfin cette aventure qui sera, sur le plan du fait anecdotique, un échec à la fois désiré et imposé, se change en une poursuite logique qui s'oppose à la morale du monde, et cependant qu'elle veut la nier, elle lui emprunte toutes ses notions, ses termes de comparaison - qui sont pleins - afin de les vider (texte, p. 92).

Et, paradoxalement, par un jeu d'aller-retour inhérent à la dynamique lectorale, l'essentiel (la "clé») se trouverait dans l'accessoire (la note):

Les mots utilisés pour ma construction perdent leur pouvoir de communication. Aussi finis, bornés que possible par leurs propres contours, je tâcherai qu'ils renvoient mal aux objets qu'ils nomment, que de ces objets ne demeure captive que la plus fantomale apparence, mais que le vocable se colore de mes angoisses, et que, du rapport de chacun d'eux, tombeau sans contenu, surgisse une construction abstraite ayant force et signification (note 1, p. 84).

On le voit, l'enjeu que représente la note ne manque pas d'être paradoxal puisque la cohérence du texte dépend des ruptures et des contraintes qu'elle lui impose.

\section{Note 3}

Vingt-trois notes de longueur variable - de quelques lignes à deux pages - jalonnent sur une superficie totale de 319 pages le Traité des courtes merveilles de Vaclav Jamek. Le livre constitue, selon la description de la quatrième de couverture, une «Sorte d'autobiographie intellectuelle et d'examen de conscience d'un Tchèque de quarante ans [...] sans complaisance avec lui-même [qui] évoque son inaptitude fondamentale au bonheur [...], ses déboires d'homosexuel [...], ses démêlés avec la maison d'édition pragoise où il travaille [etc.]».

La pratique de la note surprend ici par la nature de son emplacement et de sa technique d'appellation. Elle illustre en quelque sorte le règne interventionniste de la glose interlinéaire telle que la pratiquait Montaigne, une idée, parfois un mot, appelant les autres: "Nos opinions s'entent les unes sur les autres. La première sert de tige à la seconde, la 
seconde à la tierce » ${ }^{14}$. Pas d'intervention de chiffres ou de lettres, seulement un astérisque qui annonce, dès la fin de la ligne où il apparaît, une interruption dans la linéarité du texte en cours, causée par interférence ou intrusion d'un texte parasite matériellement circonscrit par deux traits horizontaux et un élargissement de la marge, sans plus. Cette génération spontanée apparemment produite par association d'idées* s'appuie sur des considérations personnelles d'ordre référentiel, sociopolitiques ou linguistiques par exemple, que justifient la nationalité de l'auteur et le fait que le livre a été écrit directement en français.

*Par exemple:

[...] moi qui tant de fois me suis démené avec la tentation de tout lâcher, de me coucher dans le caniveau * et de rester là,

* J'aime bien ce mot, il me fait penser à un chien perclus de tristesse mais qui mord encore, de temps en temps dans le vide, il me fait penser aussi à "canevas", me désignant la trame d'un poème comme le dispositif d'une assomption, d'une ouvraison à même l'abîme; par contre, je consentirais difficilement à me coucher dans une rigole, ce serait trop grotesque, ce serait vraiment la fin.

en attendant de me résorber en glaise et gadoue. (p. 15)

L'astérisque prend ici valeur de signal revendiquant la validation de la digression qu'il introduit: une bifurcation qui, à la manière parenthétique, apporte une dérive, une citation, un complément d'information, une explication, un développement, un renforcement ou un prolongement, en tout cas une brisure dans le déroulement général. En regard du livre dans son ensemble, divisé en quatre chapitres denses, ces brisures constituent visuellement autant de pauses et, sur le plan du contenu, autant d'ouvertures.

Il reste que, en regard du contenu, face à cette sorte de greffon accidentel qui s'impose d'autorité avec l'impertinence

14 Michel de Montaigne, Essais III, 13, Paris, coll. "Le livre de poche ", 1965 , p. 313. 
54

d'un aparté, le lecteur se trouve confronté à un choix draconien: capituler (et fermer le livre) ou obtempérer, tels semblent être les termes du contrat. La lecture en différé, facultative, que propose la note infrapaginale ou finale est ici exclue au profit de l'adhésion sans condition et de la consommation immédiate.

C'est dire qu'à ce jeu la qualité de l'écriture doit être assez forte pour retenir l'attention et endiguer l'exaspération du lecteur. Le texte y parvient en ajoutant de surcroît l'originalité des points de vue qu'il offre, qu'il s'agisse de politique ou de cinéma par exemple. Il tire même profit de l'incongruité du propos hors de propos, évoquant ainsi un souvenir de jeunesse: "ce qui m'attire dans un match de football, par exemple, c'est la fiction circonscrite - donc, contenue - de la structuration produite par les sillonnements du désir» (en note, p. 108). De fait, n'y a-t-il pas, dans ce vagabondage et cette façon de prendre et de perdre son temps en marge du droit fil du récit, le reflet d'une dispersion antérieure à la structuration du désir de raconter? Cette insécurité structurelle n'est-elle pas la métaphore des relations suggérées par ces "courtes merveilles"? Comme si, en appelant à une lecture régressive, l'écriture digressive, signe extérieur d'une communication défectueuse, frayait la voie à un mode de perception plus intuitif.

L'indifférenciation du monde dans lequel on se situe et le monde dont on parle, la confusion entre texte et commentaire, entre narration et conversation qu'instaure cette procédure de dislocation du texte au profit de l'incrustation du commentaire auctorial mettent en cause, une fois de plus, la fonction phatique du discours. Dans la mesure où l'auteur en malmenant la linéarité de l'écrit mime le flot du langage et semble ainsi se livrer aux confidences à bâtons rompus, n'êprouve-t-il pas en effet l'indulgence et la complicité du lecteur?

À moins de s'inscrire dans une esthétique de la négativité, cet usage de la note - le moins raffiné qui soit en terme d' "appareil» si on le compare à la gamme des possibles offerts en la matière - demeure énigmatique. Faut-il y lire l'urgence d'une pensée bridée qui enfin trouve à exprimer librement et en sécurité, à l'abri de la langue étrangère, sa dualité? On pourrait multiplier hypothèses et interprétations, le sens en est sans doute à lire dans le texte. Celui-ci 
est trop subtil pour que ne s'y trouve pas la réponse à cette anomalie. On s'en tiendra ici au constat qui assimile cette pratique de la note à la digression.

Quel que soit l'enjeu de la nature et de l'usage des notes dans ce Traité de toute façon irréductible à l'explication strictement mécaniste, il demeure en effet que ce texte remet d'actualité, avec acuité, la question de la digression. Quignand 15 par ailleurs la pratique délibérément, et Ransmayr ${ }^{16}$ la désigne nommément en l'intégrant dans le texte.

Signe des temps? Ce retour marginal de ce que l'histoire désigne tantôt comme comble du raffinement tantôt comme comble de la grossièreté ${ }^{17}$, selon que la note et ses avatars sont perçus sur le mode de l'expansion ou de la complication, semble confirmer l'instabilité du texte. Que la présence de la note dans le texte littéraire témoigne d'un malaise, comme semblent le désigner la trace de dénégation décelée dans deux des exemples observés et le dysfonctionnement caractéristique du troisième, n'ajoute rien à ce que l'on sait déjà du mal d'être que révèle l'acte d'écriture. Elle ne fait que le souligner. En revanche, la plus-value de sens qui en résulte incite, face à la prolifération généralisée de l'écrit, à continuer de s'interroger sur la signification de cette pratique. La sollicitation qu'elle impose à la vigilance du lecteur ne cherche-t-elle pas, par le biais du nécessaire mouvement qu'elle exige, à stimuler l'acte de lecture? Si l'on croit que celui-ci dépasse le simple décodage et l'information et que le plaisir "émane d'un lien personnel entre le lecteur lisant et le texte comme tel $* 18$, la présence de la note dans le texte littéraire n'a d'étonnant que sa relative rareté.

15 " Je découvre que je ne parle plus d'Albucius. Ce chapitre est une soudaine digression. Je ne romps pas cette digression. Je noue. " (Albucius, p. 72).

16 "Première digression/Le passage du nord-est ou la route blanche des Indes - Reconstitution d'un rêve." (Les effrois de la glace et des ténébres, p. 41) suivi de: "Deuxième digression/Les chercheurs de passages - Extrait de la chronique de l'échec" (p. 76), assortie d'une: "Note complémentaire: les vainqueurs " (p. 77 ).

17 Michel Charles, * Digression, régression ", Poétique, $n^{\circ} 40$, novembre 1979 , p. 397.

18 Paul Zumthor, Performance, réception, lecture, coll. «L'Univers des discours ", Longueuil, Le Préambule, 1990, p. 27. 